curves. Cumulative TB rates were expressed as time after UK entry and time after GP registration and compared between immigrant subgroups stratified by WHO incidence in country of origin $(150-499 / 100000$ or $500+/ 100000)$ and age group at time of registration $(<16,16-35$ or $=36$ years). The number needed to screen was calculated using an overall prevalence estimate of $25 \%$ IGRA positivity, with all cases occurring in this subgroup.

Results 564 cases were recorded in 34764 immigrants. The median (IOR) observation was 2198 (982-3329) days after UK entry and 956 (358-1888) days after GP registration. There was no difference in risk with time after UK entry or GP registration and the TB rate rose linearly over 10 years. In our cohort, the 5 -year cumulative TB rate was significantly higher for immigrants from regions with incidence of 150-499 than those from 500+. The TB rate was also significantly higher in adults than children, and highest in adults aged 16-35 years (Abstract P55 table 1). For this age band, the estimated number needed to screen $(95 \% \mathrm{CI})$ with IGRAs to identify one immigrant developing TB in 5 years was 78.8 (73.2-85.2) persons.

Abstract P55 Table 1 Five-year TB rate after GP registration in immigrant subgroups

\begin{tabular}{|c|c|c|c|c|c|}
\hline & \multicolumn{2}{|c|}{ WHO incidence in country of origin } & \multicolumn{3}{|c|}{ Age at GP registration } \\
\hline & $150-499$ & $500+$ & $0-15$ & $16-35$ & $36+$ \\
\hline $\begin{array}{l}5 \text { year rate (SE)/ } \\
100000 \text { person } \\
\text { years }\end{array}$ & / 1421 (76.8) & $1045(193.7)$ & $493(98.7)$ & $1691(104)$ & 1347 (151) \\
\hline
\end{tabular}

Conclusions A new immigrant screening model using the Flag 4 GP registry and IGRA testing may be effective for identifying at-risk immigrants.

\section{P56 TREATMENT OF MULTIDRUG RESISTANT TUBERCULOSIS: WHERE ARE THE GUIDELINES FOR MONITORING?}

doi:10.1136/thoraxjn--2011-201054c.56

${ }^{1} \mathrm{~J} \mathrm{~L} \mathrm{Keal,}{ }^{2} \mathrm{H}$ Khachi, ${ }^{1} \mathrm{E}$ Hanzaree, ' V L C White. ${ }^{1}$ Department of Respiratory Medicine, Barts and the London NHS Trust, London, UK; ${ }^{2}$ Respiratory Pharmacy Department, Barts and the London NHS Trust, London, UK

Introduction Complex treatment strategies are necessary to tackle multi-drug resistant tuberculosis (MDR-TB). Adverse drug reactions (ADRs) are well documented but currently there are very few guidelines for drug monitoring aimed specifically at the durations of treatment used in MDR-TB. ${ }^{1}$

Methods We retrospectively reviewed all MDR-TB patients seen at our Trust over the last 5 years to determine which ADRs were most common and how they are assessed. We examined epidemiological factors (age, sex, ethnicity, and duration in UK), drug regimens, duration of treatment, treatment completion, ADR monitoring, and ADRs reported. All patients had baseline blood tests, visual acuity and Ishihara plates.

Results 18 patients were reviewed ( 12 male) with a mean age of 34.4 (range 10-80) years. One was HIV positive. Thirteen had lived in the UK for $<10$ years $(2>10$ years). Three were born in the UK (1 white ethnicity). Treatment regimens included a combination of 4-5 drugs selected from first line oral agents (pyrazinamide or ethambutol), $2^{\text {nd }}$ line oral bacteriostatics (PAS, prothionamide, cycloserine), a fluoroquinolone (moxifloxacin or ofloxacin), drugs which have an unclear role, (clarithromycin) and an aminoglycoside used during the first intensive phase. Planned duration of treatment for all patients was 24 months. Nine completed 2 years, two completed $>2$ years, six are on-going and one died. Reported ADRs included nausea and vomiting (38.9\%), visual impairment (38.9\%), hearing loss $(27.8 \%)$, tinnitus (27.8\%), hypothyroidism $(16.7 \%)$, arthralgia (16.7\%), hepatic impairment $(11.1 \%)$, renal impairment $(5.6 \%)$ and also fatigue, headache, rash, pruritis and dizziness. ADRs were detected through a combination of self-reporting of symptoms and direct questioning. Appropriate blood tests and drug levels were taken regularly but not following a recognised protocol.

Conclusions MDR-TB treatment regimens use toxic drugs over long periods. We support the development of a series of systematic evidence-based protocols for assessing ADRs and organisation of monitoring tests in MDR-TB patients which can be used by both physicians and specialist nurses. This should include baseline screening, regular blood tests, audiometry and visual testing as well as drug level monitoring.

\section{REFERENCE}

1. Treatment of Tuberculosis: Guidelines. 4th ed. Geneva: World Health Organization, 2009 (WHO/HTM/TB/2009.420)

\section{P57 THE AWARENESS, PERCEPTIONS AND ATTITUDES AMONG MIGRANTS TOWARDS TB SCREENING}

doi:10.1136/thoraxjnl-2011-201054c.57

${ }^{1}$ Y Y S Ho, ${ }^{2} \mathrm{D}$ Nazareth, ${ }^{2} \mathrm{~J}$ Gallagher, ${ }^{2} \mathrm{~S}$ Kazmi, ${ }^{2} \mathrm{P} \mathrm{D} O \mathrm{Davies} .{ }^{1}$ University of Liverpool, Liverpool, UK; ${ }^{2}$ Liverpool Heart and Chest Hospital, Liverpool, UK

Introduction There is a wide variation in the provision of $\mathrm{TB}$ screening services for migrants worldwide. New entrant screening for TB helps to identify and treat active and latent TB at an early stage, reduce risk of transmission and identifies those at a high-risk. It is common practice to screen new entrants from countries where the prevalence is high. However, it is sometimes perceived that screening for immigrants can be stigmatising. In addition, there is a poor understanding of this disease and large cultural differences in perceptions exist. This study explores the different attitudes towards screening and the current awareness, perceptions and attitudes towards $\mathrm{TB}$, in order to tailor the current screening programme to different cultural backgrounds.

Methods 27 participants (63\% male) were recruited at the weekly multi-disciplinary Liverpool TB contact-tracing clinic. Semi-structured interviews (18\% interpreter help) exploring the attitudes towards screening and the disease were conducted.

Results $59 \%$ of participants were aware of the causative agent, $74 \%$ identified at least 1 associated symptom and $89 \%$ recognised that transmission was air borne. $89 \%$ considered TB curable, 33\% were aware of the link with HIV and $44 \%$ stressed that a greater awareness of TB among the general public is needed. The main source of information about the disease was family and friends (43\%) and clinic (42\%). The majority (59\%) felt there was a stigma attached with fear of social isolation and an impact on occupation. $59 \%$ expressed a positive attitude towards screening and perceived it to be acceptable due to the availability of free treatment. The majority of the participants regarded screening as prevention of transmission and felt it was their responsibility in society. $22 \%$ of the participants thought it was unnecessary or were unsatisfied with the current screening process. Seven per cent of the participants demonstrated poor understanding of the screening process, having already undergone screening.

Conclusions The majority of the migrants found TB screening to be acceptable and demonstrated a positive attitude, although some barriers still exist. Improving TB awareness in the communities will help increase the acceptability of screening. 